\title{
CD226 opposes TIGIT to disrupt Tregs in melanoma
}

Julien Fourcade, ${ }^{1}$ Zhaojun Sun, ${ }^{1}$ Joe-Marc Chauvin,, Mignane Ka, ${ }^{1}$ Diwakar Davar, ${ }^{1}$ Ornella Pagliano, ${ }^{1}$ Hong Wang, ${ }^{2}$ Sofiane Saada, ${ }^{1}$ Carmine Menna, ${ }^{1}$ Rada Amin, ${ }^{1}$ Cindy Sander, ${ }^{1}$ John M. Kirkwood, ${ }^{1}$ Alan J. Korman, ${ }^{3}$ and Hassane M. Zarour ${ }^{1,4}$

'Department of Medicine and Division of Hematology/Oncology and 2Department of Biostatistics, University of Pittsburgh, School of Medicine, Pittsburgh, Pennsylvania, USA. ${ }^{3}$ Bristol-Myers Squibb, Biologics Discovery California, Redwood City, California, USA. ${ }^{4}$ Department of Immunology, University of Pittsburgh, School of Medicine, Pittsburgh, Pennsylvania, USA.

CD4 ${ }^{+}$Tregs impede T cell responses to tumors. They express multiple inhibitory receptors that support their suppressive functions, including T cell Ig and ITIM domain (TICIT). In melanoma patients, we show that Tregs exhibit increased TIGIT expression and decreased expression of its competing costimulatory receptor CD226 as compared with CD4+ effector T cells, resulting in an increased TICIT/CD226 ratio. Tregs failed to upregulate CD226 upon T cell activation. TICIT+ Tregs are highly suppressive, stable, and enriched in tumors. TICIT and CD226 oppose each other to augment or disrupt, respectively, Treg suppression and stability. A high TIGIT/CD226 ratio in Tregs correlates with increased Treg frequencies in tumors and poor clinical outcome upon immune checkpoint blockade. Altogether, our findings show that a high TIGIT/CD226 ratio in Tregs regulates their suppressive function and stability in melanoma. They provide the rationale for novel immunotherapies to activate CD226 in Tregs together with TICIT blockade to counteract Treg suppression in cancer patients.

Conflict of interest: The authors have declared that no conflict of interest exists.

Submitted: March 19, 2018

Accepted: June 12, 2018

Published: July 25, 2018

\section{Reference information:} JCI Insight. 2018;3(14):e121157. https://doi.org/10.1172/jci. insight.121157.

\section{Introduction}

Tregs, which express the transcription factor Foxp3, regulate innate and adaptive immune cells and maintain self-tolerance (1). In preclinical cancer models, Tregs potentially abrogate $\mathrm{T}$ cell responses to tumors (2). In humans, Tregs are highly enriched in many solid tumors, including primary melanoma $(3,4)$, invaded lymph nodes (5), and metastatic melanoma (MM) (4-6). High-frequency Tregs correlate with tumor progression and poor survival in many solid tumors, including melanoma (7), and have been associated with poor clinical outcome in melanoma patients (MPs) treated with immunotherapy (8). Tregs encompass multiple $T$ cell subsets that control immunity through a variety of mechanisms depending on various environmental cues. In particular, Tregs express multiple inhibitory receptors, including CTLA-4, PD-1, Tim-3, and T cell Ig and ITIM domain (TIGIT), which promote their development and suppressive functions (9-13). TIGIT is expressed not only by Tregs but also by activated $\mathrm{T}$ and NK cells $(11,14)$. TIGIT binds with high and low affinity to PVR/CD155 and Nectin-2/CD112, respectively, which are expressed on monocytes, dendritic cells, and tumor cells, including melanoma $(15,16)$. Interestingly, TIGIT competes with the costimulatory counter-receptor DNAM-1/CD226 (17), which binds to the same ligands with lower affinity $(11,18,19)$. Whereas CD226 enhances T cell activation $(17,20)$, TIGIT inhibits T cells through T cell-intrinsic inhibitory effects (21), IL-10 production by dendritic cells upon PVR ligation (11), or competition with CD226 for PVR binding (22). In mice and humans, TIGIT is upregulated and coexpressed with PD-1 by CD8 ${ }^{+}$tumor-infiltrating (Ti) lymphocytes (TILs), and dual PD1/TIGIT blockade enhances potent antitumor $\mathrm{CD}^{+} \mathrm{T}$ cell responses and tumor regression $(16,23)$. In contrast to TIGIT, there is ample evidence that CD226 promotes antitumor immune responses mediated by NK and T cells $(17,18,24,25)$ Several lines of evidence support the role of TIGIT in regulating Treg-mediated suppression in animals and humans. The TIGIT locus is hypomethylated in human Tregs and binds to Foxp3 (26). In mice and healthy donors (HDs), TIGIT $^{+}$Tregs represent a subset of activated and highly suppressive Tregs that selectively inhibit Th1 and Th17 responses (12). Upon activation with an agonistic anti-TIGIT antibody, Tregs displayed increased production of fibrinogen-like protein 2 (FGL2), which promoted TIGIT $^{+}$Treg-mediated suppression (12). In mice with B16 melanoma, TIGIT is upregulated by highly suppressive Ti Foxp $3^{+}$ 
Tregs and acts primarily in Tregs to dampen antitumor immunity (27). In addition, CD226 ${ }^{+}$TIGIT- $^{-}$Tregs exhibit reduced demethylation of the Foxp3 gene locus at the Treg-specific demethylated region (TSDR) ex vivo and lower suppressive capacities following in vitro expansion (28). However, the role of CD226 in modulating Treg functions remains unknown. Here, we report that CD25 $5^{\text {hiFoxp }} 3^{+}$Tregs in HDs and MPs expressed high-level TIGIT and low-level CD226 as compared with CD4 ${ }^{+}$effector T cells (Teffs). Tregs in tumors further upregulated TIGIT and downregulated CD226, resulting in a higher TIGIT/CD226 ratio. TIGIT and CD226 exerted opposite effects in Tregs upon PVR binding. TIGIT acted in Tregs to augment their suppression, while CD226 disrupted their suppression and stability. A high TIGIT/CD226 ratio in Tregs associated with higher Treg frequencies in tumors and poor clinical outcome upon immune checkpoint blockade (ICB).

\section{Results}

Tregs exhibit a high TIGIT/CD226 expression ratio in the periphery and at tumor sites of MPs. We first evaluated TIGIT expression by Tregs and non-Tregs ex vivo in peripheral blood mononuclear cells (PBMCs) of HDs and MPs and MM TILs. CD25 $5^{\text {hi }}$ oxp $3^{+}$Treg frequencies were higher in MM TILs (mean frequency, $7.4 \% \pm \mathrm{SD} 5.9 \%)$ than in PBMCs of MPs $(1.6 \% \pm 1.2 \%)$ and HDs $(1.7 \% \pm 0.8 \%)$ (Figure $1 \mathrm{~A}$ and Supplemental Figure 1A; supplemental material available online with this article; https://doi.org/10.1172/jci. insight.121157DS1). CD25 ${ }^{\mathrm{hi}}$ Foxp3 ${ }^{+}$Tregs in PBMCs of HDs and MPs expressed higher TIGIT (HD: mean frequency, $77.1 \% \pm 7.4 \%$; mean MFI, $1,510 \pm 500$; MP: mean frequency, $83.7 \% \pm 14.1 \%$; mean MFI, 1,903 \pm 744 , respectively) than $\mathrm{CD}_{2} 5^{+}$Foxp3 $^{-}$(HD: mean frequency, 21.6\% \pm 5.8\%; mean MFI, $313 \pm 120$; MP: mean frequency, $26.6 \% \pm 12.4 \%$; mean MFI, $410 \pm 214$, respectively) and CD25-Foxp3- (HD: mean frequency, 15\% $\pm 6.1 \%$; mean MFI, $233 \pm 115$; MP: mean frequency, $18.3 \% \pm 6.5 \%$; mean MFI, $286 \pm 150$, respectively) $\mathrm{CD}^{+}$Teffs as well as total $\mathrm{CD}^{+} \mathrm{T}$ cells (HD: mean frequency, $18.8 \% \pm 5.3 \%$; mean MFI, $293 \pm 107$; MP: mean frequency, 21.4\% \pm 6.9\%; mean MFI, $383 \pm 121$, respectively) (Figure 1, A and B). In MM, CD25 $5^{\text {hiFoxp3 }}{ }^{+}$Tregs expressed higher TIGIT (mean frequency, 99.1\% $\pm 1.6 \%$ and MFI, 6,119 \pm 4,481 ) than CD25 ${ }^{+}$Foxp3 $^{-}$(mean frequency, $65 \% \pm 16.6 \%$ and MFI, 1,766 $\pm 1,292$ ), CD25-Foxp3- (mean frequency, $35.5 \% \pm 11.2 \%$ and MFI, $491 \pm 372$ ), and total (mean frequency, $53.2 \% \pm 12.6 \%$ and MFI, $1,568 \pm 1,447$ ) CD $4^{+}$TILs (Figure 1, A and B). Tregs exhibited higher TIGIT expression in MM TILs than in PBMCs of MPs and HDs (Figure 1, A and B). We also observed higher TIGIT expression by Tregs than CD8 ${ }^{+}$TILs in MM (Supplemental Figure 1B).

Because TIGIT competes with CD226 for binding to the same ligands, we next wanted to assess CD226 expression by Tregs of HDs and MPs. Circulating CD25 $5^{\text {hi }}$ oxp $3^{+}$Tregs in PBMCs of HDs and MPs exhibited lower CD226 expression ex vivo both in terms of frequency (mean $\pm \mathrm{SD}, 71.1 \% \pm 15.2 \%$ and $74.1 \% \pm$ 11.4\% for HDs and MPs, respectively) and MFI (1,450 \pm 743 and 1,569 \pm 406 for HDs and MPs, respectively) than CD25-Foxp3- (mean, $89.1 \% \pm 7.7 \%$ and MFI, 1,957 \pm 990 for HDs; mean, $94.6 \% \pm 2.1 \%$ and MFI, 3,268 \pm 1,464 for MPs), CD25 ${ }^{+}$Foxp3 $^{-}$(mean, $89 \% \pm 8 \%$ and MFI, 4,141 $\pm 2,080$ for HDs; mean, $90.8 \% \pm$ $5.2 \%$ and MFI, 4,477 $\pm 1,573$ for MPs) CD4 ${ }^{+}$Teffs as well as total CD4 ${ }^{+} \mathrm{T}$ cells (mean, $90.4 \% \pm 5.4 \%$ and MFI 2,669 \pm 1,401 for HDs; mean, 92.6\% \pm 3.4\% and MFI, 3,505 \pm 1,318 for MPs) (Figure 1, C and D). As a result, the ratio of TIGIT to CD226 expression was higher on CD25 hiFoxp3 ${ }^{+}$Tregs than on CD25- or CD25 ${ }^{+}$Foxp3- Teffs in PBMCs of HDs and MPs (Figure 1E). In MM, CD25 ${ }^{\text {hi Foxp3 }}{ }^{+}$Tregs exhibited lower CD226 expression (mean, $57.6 \% \pm 14.3 \%$ and MFI, 1,007 \pm 440 ) than CD25-Foxp3- (mean, $84.7 \% \pm 9.9 \%$ and MFI, 2,450 $\pm 1,067$ ), CD25 ${ }^{+}$Foxp3 $^{-}$(mean, $81 \% \pm 15.2 \%$ and MFI, 2,276 $\pm 1,249$ ), and total CD4 ${ }^{+}$TILs (mean, 80.7\% $\pm 12.2 \%$ and MFI, 2,278 \pm 828), as well as circulating Tregs in HDs and MPs (Figure 1, C and D), resulting in a higher TIGIT/CD226 ratio at tumor sites (mean ratio of the percentages of $\mathrm{TIGIT}^{+} /$ CD226 ${ }^{+}$cells: $1.7 \pm 0.6,1.2 \pm 0.3$, and $1.3 \pm 0.2$, and mean ratio MFI of TIGIT/CD226, $13.9 \pm 11.5,2.7$ \pm 1.1 , and $2.7 \pm 1.8$, for CD4+ Tregs in MM TILs and PBMCs of MPs and HDs, respectively; Figure 1E)

Collectively, as compared with Teffs, Tregs in the periphery of HDs and MPs and at tumor sites displayed higher and lower expression of TIGIT and CD226, respectively, resulting in an increased TIGIT/ CD226 ratio that is predominant in the tumor microenvironment.

Tregs fail to upregulate CD226 upon TCR activation. To investigate whether a high TIGIT/CD226 ratio in Tregs is reversed by TCR activation, Tregs were isolated from PBMCs and MM of MPs prior to in vitro stimulation (IVS) and evaluation of CD226 and TIGIT expression by flow cytometry and realtime PCR. In sharp contrast to CD25- Teffs, circulating and Ti CD25 $5^{\text {hi }}$ D $127^{-}$Tregs failed to upregulate CD226 upon TCR activation while both Tregs and Teffs upregulated TIGIT (Figure 2, A-C). Upon TCR 
A

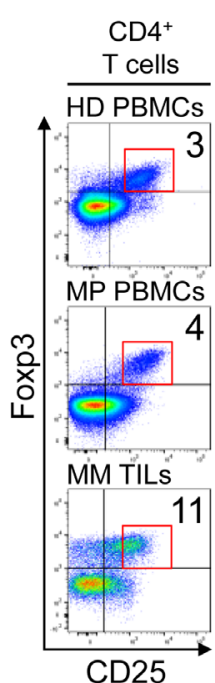

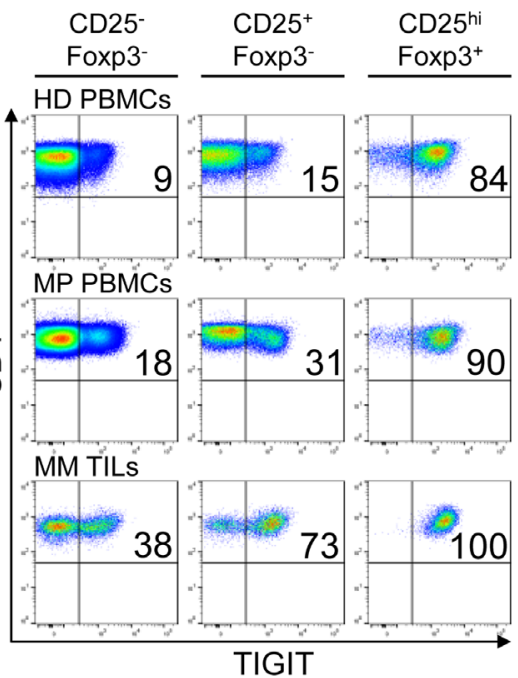

B $\diamond$ HDPBMCs $\diamond$ MP PBMCs $\bullet$ MM TILs
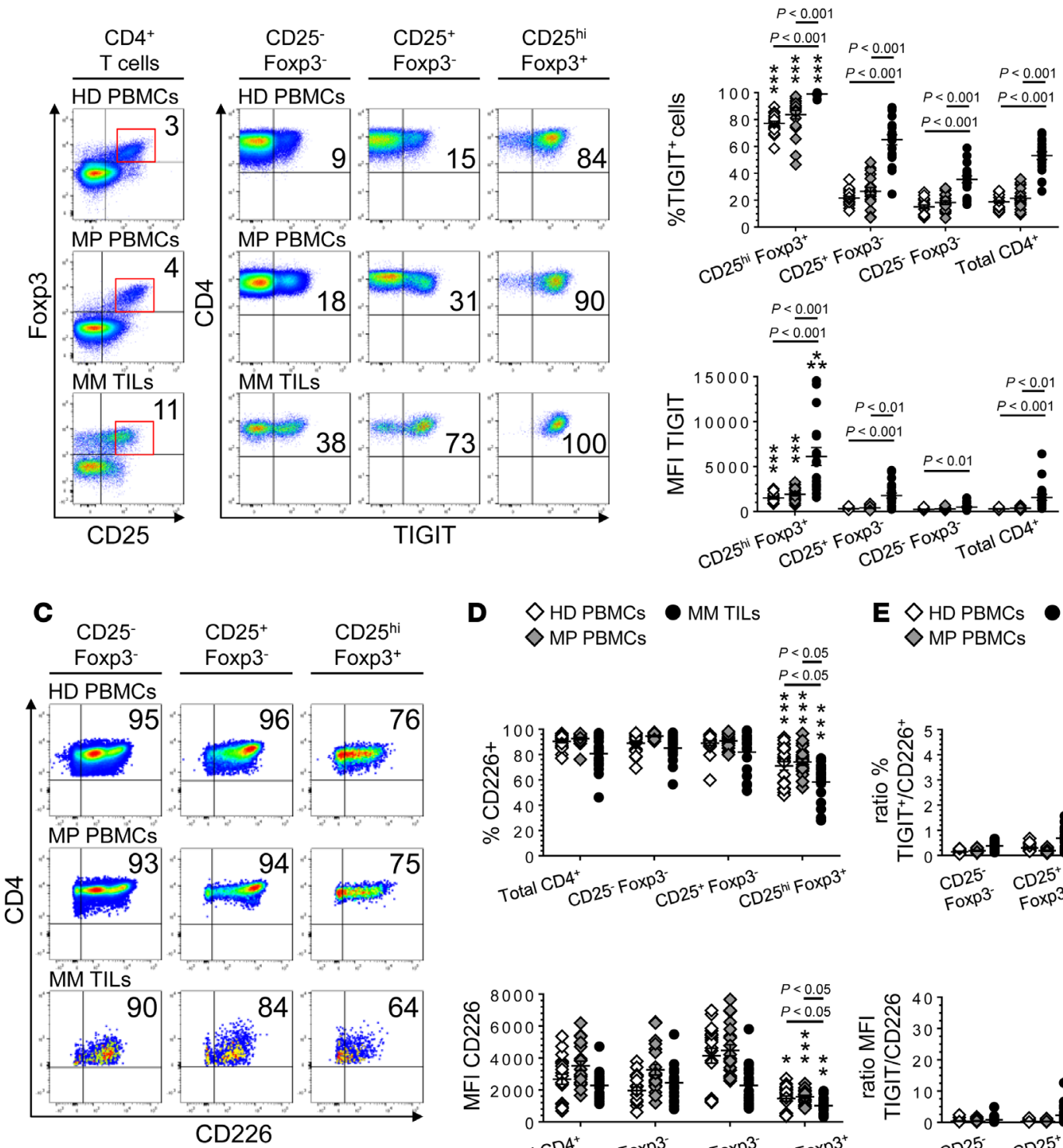

D $\quad \diamond$ HD PBMCs

- MM TILs

E $\diamond$ HD PBMCs $\bullet$ MMTILs
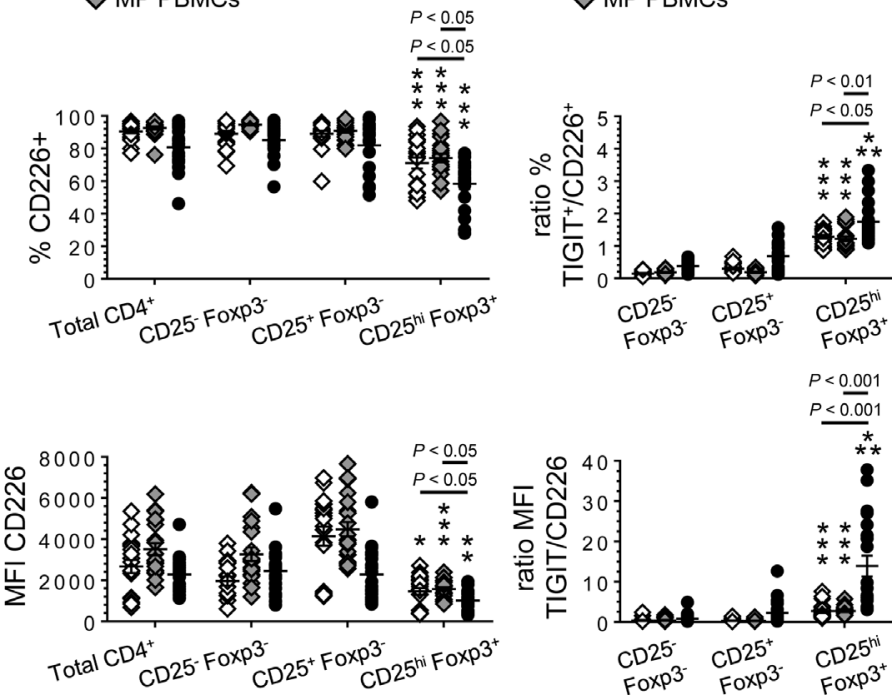

Figure 1. Tregs exhibit a high ratio of TICIT/CD226 expression in the periphery and at tumor sites of melanoma patients. (A and B) Dot plots from 3 representative donors (A) and summary data (B) showing ex vivo percentages and mean fluorescence intensity (MFI) of TICIT expression by CD25 ${ }^{\text {hi Foxp } 3^{+} \text {CD } 4^{+} \text {Tregs }}$

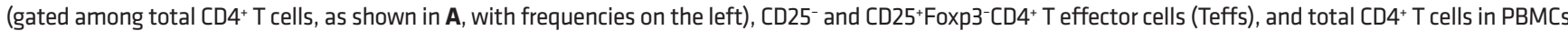
of healthy donors (HDs) and melanoma patients (MPs) and in metastatic melanoma (MM) tumor-infiltrating lymphocytes (TILs). $n=20$. (C and D) Dot plots from 3 representative donors (C) and summary data (D) showing ex vivo percentages and MFI of CD226 expression by CD25- or CD25+Foxp3-CD4 ${ }^{+}$Teffs, $C D 25^{\text {hi }}$ Foxp3 $3^{+}$ Tregs, and total CD4 ${ }^{+}$T cells in PBMCs of HDs and MPs and in MM TILs. $n=20$. (E) Summary data showing the ratio of TIGIT to CD226 ex vivo expression (percentage and MFI) by CD25- or CD25+Foxp3-CD4 ${ }^{+}$Teffs and CD25 hi Foxp3 ${ }^{+}$Tregs in PBMCs of HDs and MPs and in MM TILs. $n=20$. Results represent the mean of independent experiments. $P$ values were obtained by 1-way ANOVA and repeated-measures ANOVA followed by Tukey's test (B and D) or by Kruskal-Wallis test and Friedman's test followed by Dunn's test (E). Horizontal bars depict mean values. Error bars indicate SEM. ${ }^{*} P<0.05 ;{ }^{* *} P<0.01 ;{ }^{* * *} P<0.001$.

activation, circulating Tregs exhibited lower CD226 and higher TIGIT gene expression than Teffs, supporting that CD226 and TIGIT expression in Tregs is regulated at the transcriptional level (Figure 2D). To investigate whether TIGIT activation promotes CD226 downregulation, circulating TIGIT $^{+}$Tregs from MPs were stimulated with anti-CD3/CD28 beads and agonistic anti-TIGIT mAbs (22) prior to CD226 evaluation by flow cytometry. TIGIT activation did not affect CD226 expression, arguing against the role of TIGIT-intrinsic effects in regulating CD226 expression in Tregs (Figure 2E).

Collectively, our results show that while both Teffs and Tregs upregulate TIGIT upon TCR activation, Tregs fail to upregulate CD226, resulting in higher TIGIT/CD226 ratio. 
A

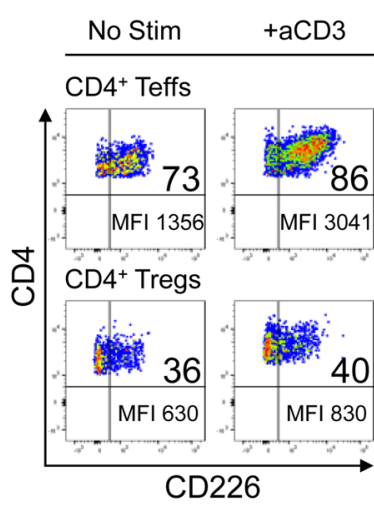

C
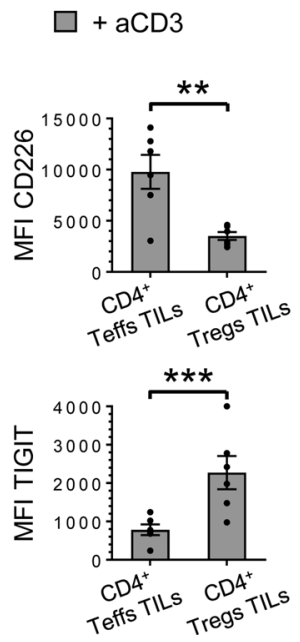

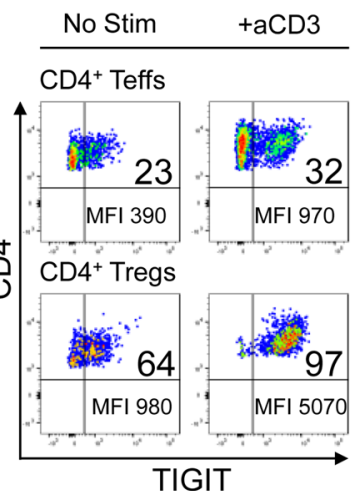

D
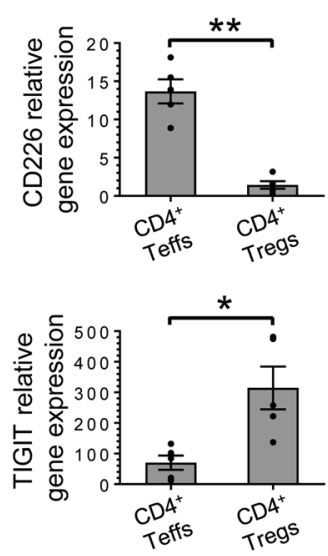

B $\quad \square$ No Stimulation

$+\mathrm{aCD} 3$

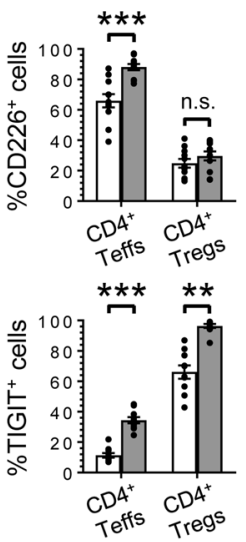

$\mathbf{E}$

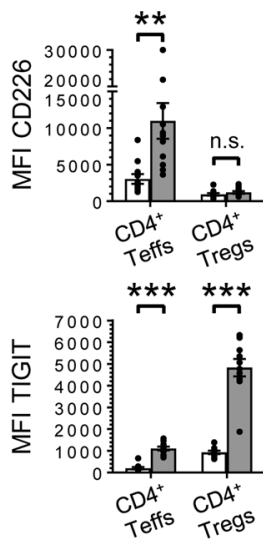

$\square+\lg G$

+ aTIGIT 318.28.2.1
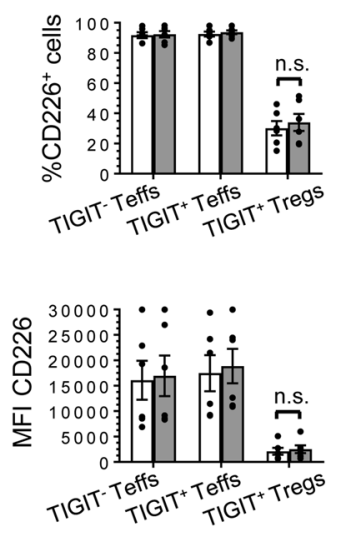

Figure 2. Tregs fail to upregulate CD226 upon TCR activation. (A and B) Dot plots from 1 representative experiment (A) and summary data (B) showing the percentages and mean fluorescence intensity (MFI) of CD226 and TICIT expression by CD25-CD4+ ${ }^{+}$effector cells (Teffs) and CD25 hicD127- Tregs isolated from PBMCs of melanoma patients (MPs) after a 6-day in vitro stimulation (IVS) with or without anti-CD3 mAbs. $n=10$. (C) Summary data showing the MFI of CD226 and TICIT expression by $\mathrm{CD}^{+}$Teffs and Tregs isolated from metastatic melanoma (MM) tumor-infiltrating lymphocytes (TILs) and stimulated as in A and B. $n=6$. (D) CD226 and TIGIT relative gene expression measured by real-time PCR in CD25-CD4+ Teffs and CD25 hiCD127-CD4+ Tregs isolated from PBMCs of MPs after a 6-day culture with anti-CD3/CD28 beads. $n=5$. (E) Pooled data showing the percentages and MFI of CD226 expression by TIGIT- and/or TIGIT+CD25-CD4+ ${ }^{+}$Teffs and CD25 hiCD127-CD4+ Tregs isolated from PBMCs of MPs after a 6-day IVS with anti-CD3/CD28 beads in the presence of agonistic anti-TICIT mAbs (clone 318.28.21) or IgG control mAbs. $n=6$. Results represent the mean of independent experiments. Error bars indicate SEM. $P$ values were obtained by paired and/or unpaired $t$ tests. ${ }^{*} P<0.05 ;{ }^{* *} P<0.01 ;{ }^{* *} P<0.001$.

TIGIT ${ }^{+}$Tregs are highly suppressive, activated, and stable in MPs. TIGIT ${ }^{+}$Tregs in HDs and mouse tumor models represent a subset of highly activated and suppressive Tregs (12, 27). In line with these studies, we observed that $\mathrm{TIGIT}^{+} \mathrm{CD} 25^{\mathrm{hi}} \mathrm{CD} 127^{-}$Tregs isolated from PBMCs of MPs exhibited higher suppression of allogeneic $\mathrm{CD} 8^{+} \mathrm{T}$ cells than TIGIT-CD25 ${ }^{\text {hi }} \mathrm{CD} 127^{-}$Tregs, whereas TIGIT- and TIGIT ${ }^{+} \mathrm{CD} 25^{-}$Teffs exhibited no suppressive activity (Figure 3A). TIGIT ${ }^{+}$Treg-mediated suppression was abolished in Transwell experiments, indicating that they act in a cell-to-cell contact-dependent fashion (Supplemental Figure 2, A and B). While TIGIT $^{+}$and TIGIT-CD25 ${ }^{\text {hi }}$ CD $127^{-}$Tregs expressed similar Foxp3 levels, TIGIT $^{+}$Tregs $^{-}$ displayed higher demethylation of the Foxp3 gene locus at the TSDR than TIGIT- Tregs, suggesting higher stability (Figure 3B). Upon IVS with anti-CD3/CD28 beads, TIGIT ${ }^{+} \mathrm{CD} 25^{\text {hi }} \mathrm{CD} 127^{-}$Tregs produced more FGL2 than TIGIT-CD25 $5^{\text {hi }}$ CD127- Tregs, whereas TIGIT $^{+}$and TIGIT- $^{-}$Tregs produced similar amounts of TGF- $\beta 1$ (Figure 3C). Both TIGIT ${ }^{+}$and TIGIT- Tregs produced very low amounts of IL-2 and IFN- $\gamma$ (Supplemental Figure 2C). As compared with circulating TIGIT ${ }^{-}$and TIGIT ${ }^{+}$CD $25^{\text {hi }}$ Foxp $^{+}$Tregs in HDs and MPs, MM TIGIT ${ }^{+} \mathrm{CD} 25^{\text {hiFoxp }} 3^{+}$Tregs expressed higher levels of molecules involved in Treg homeostasis and/or suppressive functions, such as CTLA-4 (13), CD39 (29), PD-1 (9), and Tim-3 (10) (Figure 3D), or 
A

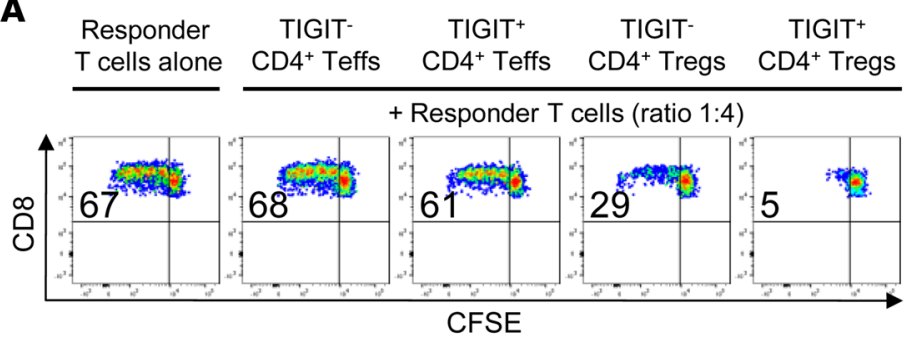

CFSE

B

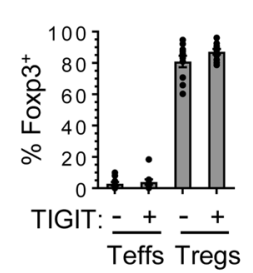

D

$\diamond$ HD PBMCs

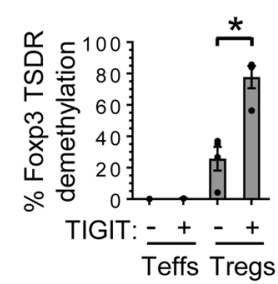

C $\square+a C D 3 / C D 28$
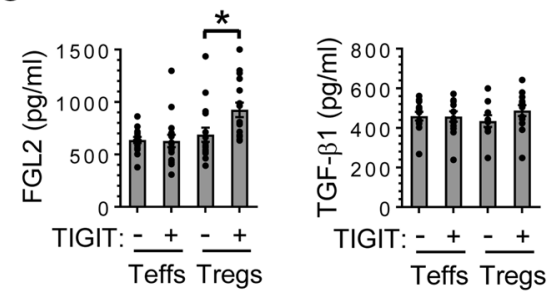

$\diamond M P$ PBMCs

MM TILs

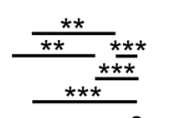

E $\diamond$ HD PBMCs

$\diamond$ MP PBMCs

MM TILS
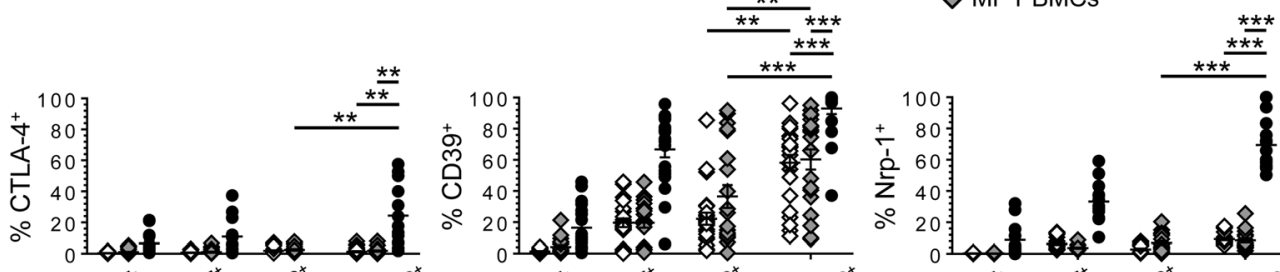

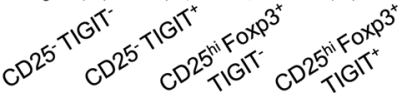
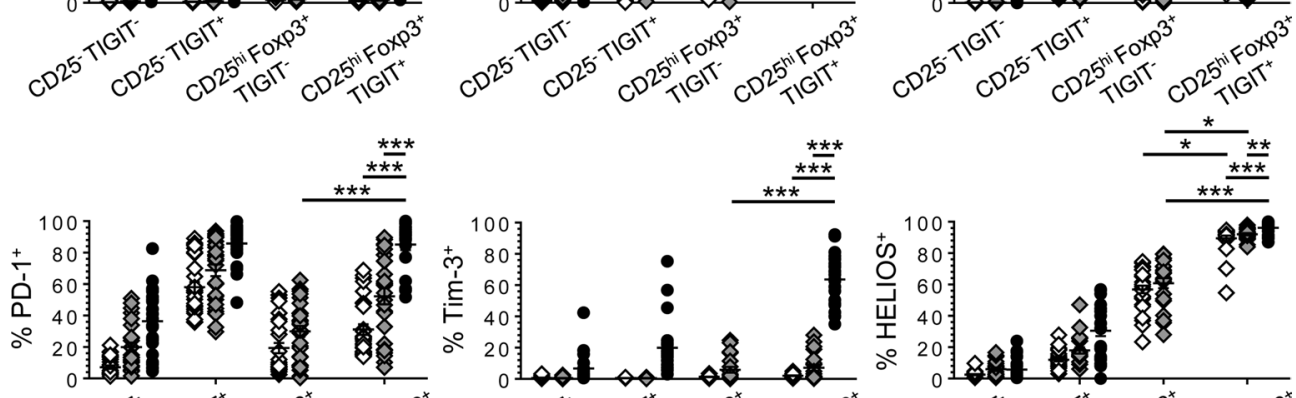

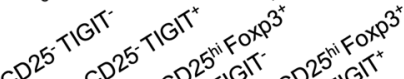
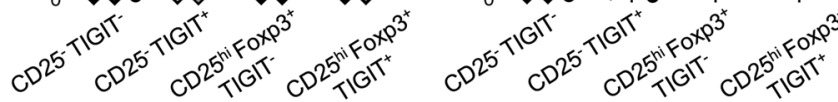

F $\diamond$ HD PBMCs

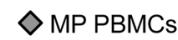

MM TILs

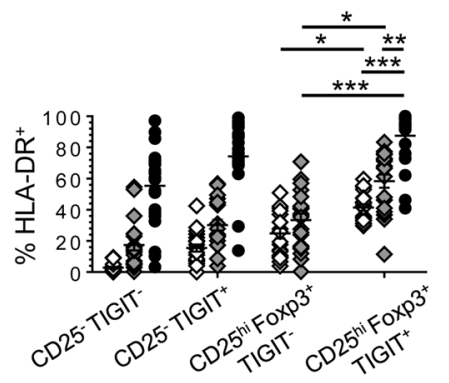

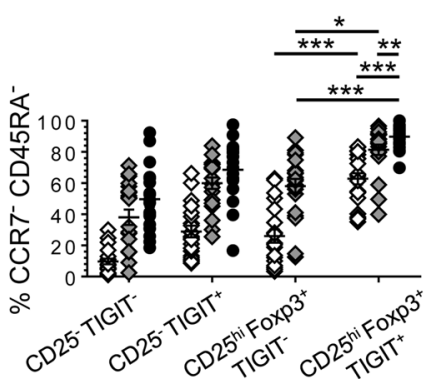

Figure 3. TIGIT $^{+}$Tregs in melanoma patients are highly suppressive, activated, and stable. (A) Flow cytometric analysis from 1 representative experiment showing the percentages of $\mathrm{CFSE}^{10}$ proliferating responder $\mathrm{CD}^{+}$ T cells after a 6-day in vitro stimulation (IVS) in the absence or presence of TICIT- or TIGIT+CD25-CD4+ ${ }^{+}$effector cells (Teffs) or CD25 hicD127- Tregs isolated from PBMCs of melanoma patients (MPs) and summary data showing the suppression of responder $\mathrm{CD}^{+} \mathrm{T}$ cell proliferation by TIGIT- or TIGIT $^{+}$Tregs at Treg-to-responder cell ratios of 1:4 $(n=8), 1: 8(n=7)$, and 1:12 $(n=4)$. (B) Pooled data showing the percentages of Foxp3 expression ( $n=$ 10) and Foxp3 Treg-specific demethylated region (TSDR) demethylation $(n=4)$ in TIGIT- $^{-}$or TIGIT+CD25-CD4+ Teffs or CD25 hiCD127- Tregs isolated from PBMCs of MPs. (C) Pooled data showing the production of FGL2 ( $n$ $=16)$ and TCF- $\beta 1(n=13)$ by TIGIT- or TICIT $^{+}$CD25-CD4 ${ }^{+}$Teffs or CD25 ${ }^{\text {hi }}$ CD127 Tregs isolated from PBMCs of MPs after a 6-day IVS with anti-CD3/CD28 beads. (D-F) Summary data showing ex vivo expression (\%) of indicated markers ( $\boldsymbol{D}$ and $\mathbf{E}$ ) and percentages of HLA-DR ${ }^{+}$and CCR7-CD45RA- cells (F) within TICIT- $^{-}$and/or TIGIT+CD25-CD4 ${ }^{+}$ Teffs and $\mathrm{CD}_{25} 5^{\text {hi }}$ Foxp $^{+}$Tregs in PBMCs of healthy donors (HDs) and melanoma patients (MPs) and in metastatic melanoma (MM) tumor-infiltrating lymphocytes (TILs). $n=20$. Results represent the mean of independent experiments. Horizontal bars depict mean values. Error bars indicate SEM. $P$ values were obtained by paired $t$ tests $(\mathbf{A}-\mathbf{C})$ and by Friedman's test and/or Kruskal-Wallis test followed by Dunn's test or by repeated-measures ANOVA followed by Tukey's test $(\mathbf{D}-\mathbf{F}) .{ }^{*} P<0.05$; ${ }^{* *} P<$ $0.01 ;{ }^{* *} P<0.001$.

in Treg stability, such as neuropilin-1 (Nrp-1) (30) and Helios (31, 32) (Figure 3E). Within circulating Tregs in HDs and MPs, TIGIT ${ }^{+}$Tregs expressed more CD39 and Helios than TIGIT- Tregs (Figure 3, D and E). Foxp $3^{+} \mathrm{CD}^{+} \mathrm{T}$ cells encompass functionally and phenotypically distinct $\mathrm{T}$ cell subsets (33). Foxp $3^{\text {hi }} \mathrm{C}$ D45RA- activated/effector Tregs exhibited higher TIGIT expression than Foxp $3^{\text {lo }} \mathrm{CD}^{-}$45A ${ }^{+}$resting Tregs and Foxp $3^{\text {lo }} \mathrm{CD} 45 \mathrm{RA}^{-}$nonsuppressive $\mathrm{CD}^{+} \mathrm{T}$ cells in the periphery and at tumor sites of MPs (Supplemental Figure 3). In addition, $\mathrm{TIGIT}^{+} \mathrm{CD} 25^{\mathrm{hi}} \mathrm{Foxp} 3^{+}$Tregs in PBMCs and TILs of MPs displayed higher frequencies of HLA-DR ${ }^{+}$and CCR7-CD45RA- effector memory cells than circulating TIGIT- ${ }^{-}$Tregs, indicating a more activated/mature phenotype (Figure $3 \mathrm{~F}$ ). 
Collectively, in MPs, TIGIT ${ }^{+}$Tregs are highly suppressive as compared with TIGIT ${ }^{-}$Tregs and display an activated and stable Treg phenotype.

PVR modulates Treg suppression in melanoma through TIGIT and CD226. TIGIT activation with an agonistic antibody appears to promote Treg suppression in HDs (12). However, whether PVR modulates Treg suppression through TIGIT and/or CD226 has not been studied yet. To address this question, we isolated TIGIT $^{+}$and/or total CD25 ${ }^{\text {hi }}$ CD127- Tregs from PBMCs and tumors of MPs and evaluated their suppressive activity on allogeneic $\mathrm{CD} 8^{+} \mathrm{T}$ cells upon stimulation with non-CD3 cells and anti-CD3 mAbs with or without anti-TIGIT or anti-CD226-blocking mAbs. No change in Treg suppression was observed in the presence of anti-TIGIT or anti-CD226 mAbs as compared with IgG control mAbs (Supplemental Figure 4 , A-C). We reasoned that the level of PVR expression on antigen-presenting cells (APCs) may be critical to activate TIGIT and CD226 on Tregs. Indeed, PVR expression on circulating APCs within non-CD3 cells used in these assays (mean percentage \pm SD, $35 \% \pm 20 \%$ for monocytes and $31 \% \pm 14 \%$ for DCs, respectively; Supplemental Figure 4D) was much lower than on APCs in MM (mean percentage \pm SD, $87.2 \%$ $\pm 8.5 \%$ for monocytes and $54.4 \% \pm 19 \%$ for DCs; Supplemental Figure $5 \mathrm{~A}$ ), as previously reported (16). Therefore, we repeated these experiments in the presence of human PVR-Fc with or without anti-TIGIT and/or anti-CD226 mAbs and/or IgG mAbs. We observed that PVR-Fc bound to Fc $\gamma$ receptors, resulting in increased PVR expression on the surface of APCs (Supplemental Figure 5, B and C). Circulating TIGIT $^{+} \mathrm{CD} 25^{\text {hi }} \mathrm{CD} 127^{-}$Tregs and $\mathrm{CD} 25^{\text {hi }} \mathrm{CD} 127^{-}$Tregs from MM exhibited lower suppression in the presence of PVR-Fc and anti-TIGIT mAbs as compared with PVR-Fc and/or IgG mAbs (Figure 4). The decrease in Treg-mediated suppression was abrogated when anti-CD226 mAbs were added to PVR-Fc and anti-TIGIT mAbs, suggesting that PVR-mediated activation of CD226 counteracted Treg-mediated suppression (Figure 4). To demonstrate that PVR acted directly on CD226 expressed by Tregs but not activated responder $\mathrm{CD}^{+} \mathrm{T}$ cells, TIGIT ${ }^{+} \mathrm{CD} 25^{\text {hi }} \mathrm{CD} 127^{-}$Tregs were preincubated with anti-CD226 mAbs (anti-CD226/TIG$\mathrm{IT}^{+}$Tregs), then washed to remove unbound $\mathrm{mAbs}$, and added into the wells containing responder $\mathrm{CD} 8^{+}$ $\mathrm{T}$ cells. In the presence of anti-TIGIT mAbs, PVR-Fc failed to reduce anti-CD226/TIGIT ${ }^{+}$Treg-mediated suppression (Figure 4B, bottom). In contrast to the periphery, Tregs isolated from MM exhibited higher suppressive capacities in the presence of PVR-Fc and anti-CD226 mAbs as compared with PVR-Fc and/ or IgG mAbs, and PVR-Fc and anti-TIGIT plus anti-CD226 mAbs, supporting that PVR binding to TIGIT that is highly upregulated by Ti Tregs promotes their suppressive functions (Figure 4, C and D).

Altogether, our findings show that TIGIT and CD226 interact with PVR to exert opposite effects in Tregs, augmenting or counteracting, respectively, Treg-mediated suppression.

PVR impedes Treg stability through CD226 in MPs. We next wanted to investigate whether PVR acts through CD226 to impede Treg stability. We first evaluated the effects of PVR binding to CD226 on Foxp3 expression by Tregs. TIGIT ${ }^{+} \mathrm{CD} 25^{\text {hi }} \mathrm{CD} 127^{-}$Tregs exhibited decreased Foxp3 expression upon 6-day IVS with anti-CD3/CD28 beads coated with human PVR-Fc (anti-CD3/CD28/PVR beads), but not isotype control $\mathrm{mAbs}$ (anti-CD3/CD28/IgG beads), and anti-TIGIT mAbs, as compared with IgG mAbs (Figure 5A). We also observed an increased methylation of the TSDR of the Foxp3 locus in TIGIT $^{+}$CD25 ${ }^{\text {hi }}$ CD 127- Tregs upon 6-day IVS with anti-CD3/CD28/PVR beads and anti-TIGIT mAbs as compared with anti-CD3/CD28/IgG beads and IgG mAbs (Figure 5B). These effects were abolished when anti-CD226 mAbs were added to the wells (Figure 5, A and B). Because Treg instability is associated with the acquisition of effector-like functions, such as the production of proinflammatory cytokines $(34,35)$, we next investigated the effects of PVR on cytokine production by Tregs. Circulating TIGIT $^{+} \mathrm{CD} 25^{\text {hi }} \mathrm{CD} 127^{-}$Tregs and Ti CD25 ${ }^{\text {hi }} \mathrm{CD} 127^{-}$Tregs produced more IL-2 upon stimulation with anti-CD3/CD28/PVR beads and anti-TIGIT mAbs as compared with anti-CD3/CD28/PVR and/or anti-CD3/CD28/IgG beads and IgG mAbs (Figure 5C). These effects, although modest, were abolished by the addition of anti-CD226 mAbs to the wells (Figure 5C). In addition, IFN- $\gamma$ production by Tregs from PBMCs and MM TILs was diminished by CD226 blockade upon stimulation with antiCD3/CD28/PVR beads (Supplemental Figure 6A). The production of FGL2 and TGF- $\beta 1$ by circulating and Ti Tregs did not change significantly upon stimulation with anti-CD3/CD28/PVR beads with or without anti-TIGIT mAbs with or without anti-CD226 mAbs (Supplemental Figure 6B).

Altogether, our findings show that PVR acts directly on CD226 in Tregs to decrease Foxp3 expression and promote the acquisition of effector-like functions, disrupting Treg stability.

A high TIGIT/CD226 ratio in Tregs in MM correlates with high-Treg frequencies and poor clinical outcome upon $I C B$. To investigate the in vivo relevance of our findings in vitro, we next assessed whether a high TIGIT/ 
A
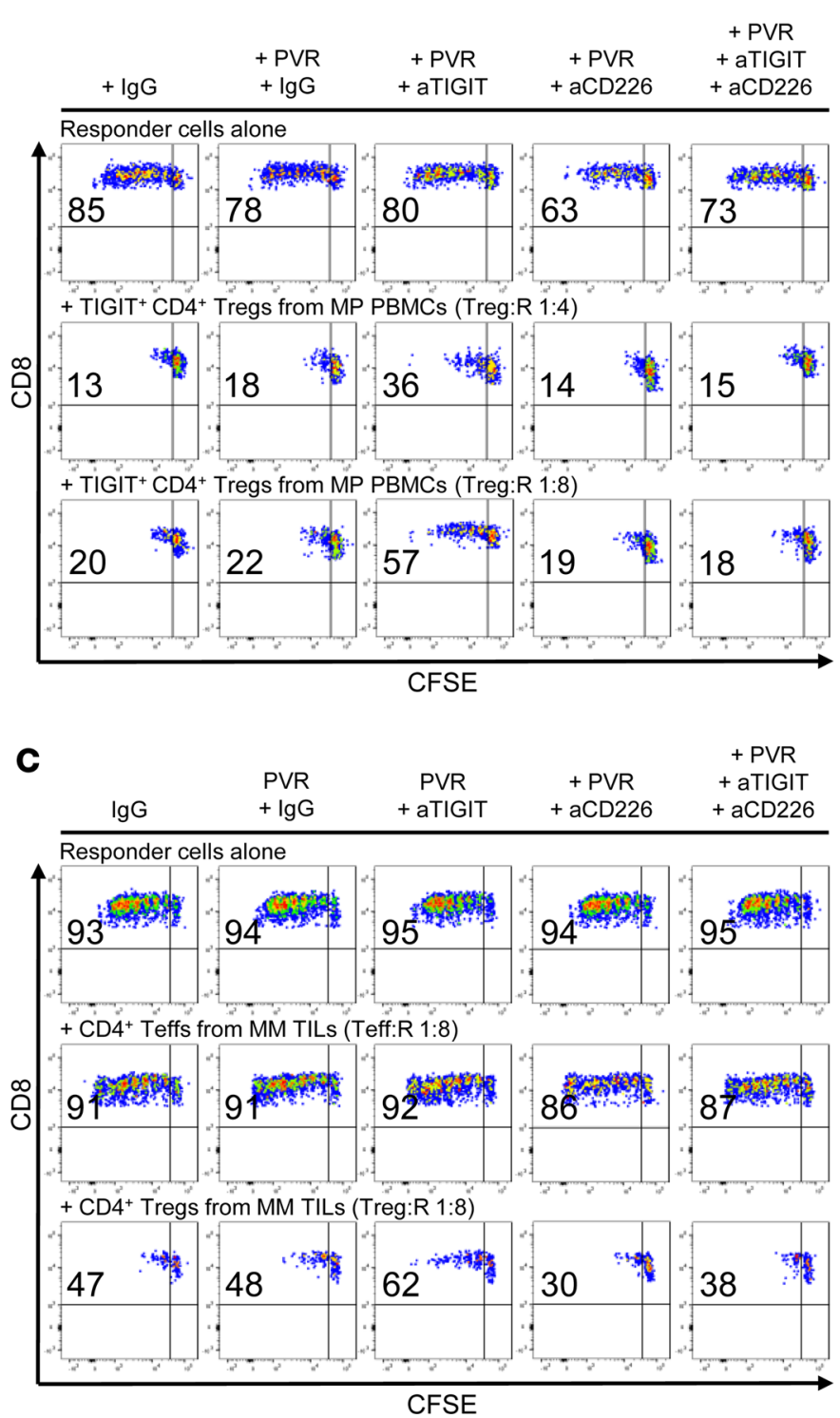
B $\mathbb{Q} \lg G \quad \square P V R+a C D 226$
$\square P V R+\lg G \quad \mathbb{P} V R R+a T I G I T+a C D 226$
$\square$ PVR + aTIGIT

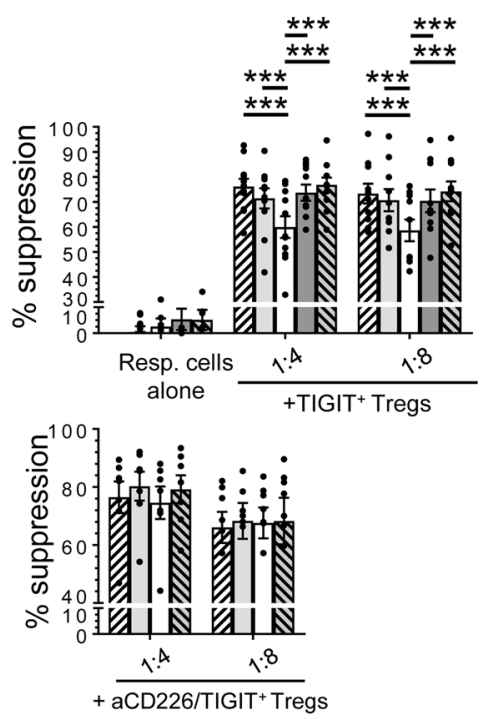

D

$\mathbb{I g} \mathrm{g}$

$\square \mathrm{PVR}+\lg \mathrm{G}$

$\square P V R+a T I G I T$

$\square \mathrm{PVR}+\mathrm{aCD} 226$

\$ PVR + aTIGIT + aCD226

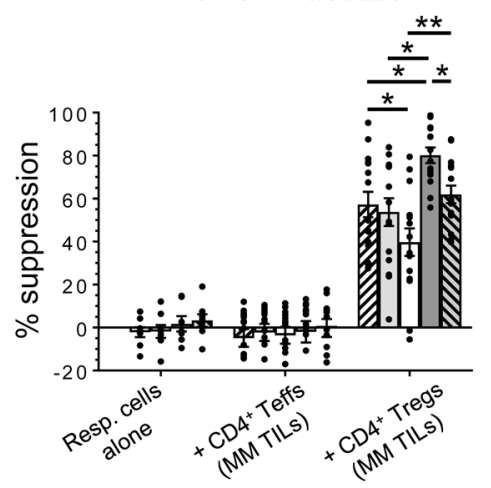

Figure 4. PVR regulates Treg suppression in melanoma through TIGIT and CD226. (A and B) Flow cytometric analysis from 1 representative experiment showing the percentages of $\mathrm{CFSE}{ }^{10}$ proliferating responder $\mathrm{CD} 8^{+} \mathrm{T}$ cells $(\mathbf{A})$ and summary data showing the suppression of responder $\mathrm{CD} 8^{+} \mathrm{T}$ cell proliferation (B) after a 6-day in vitro stimulation (IVS) with autologous antigen-presenting cells (APCs) and anti-CD3 mAbs in the absence or presence of TIGIT+CD25 hiCD127-CD4+ Tregs isolated from PBMCs of melanoma patients (MPs) and with PVR-Fc and/or anti-TIGIT and/or anti-CD226-blocking mAbs and/or lgG control mAbs. Ratios of Tregs to responder cells are indicated. CD4 ${ }^{+}$Tregs were used either untouched ( $\mathbf{A}$ and $\mathbf{B}$, top; $\left.n=10\right)$ or were separately preincubated with anti-CD226 mAbs (aCD226/TIGIT+ Tregs) before thorough washing and plating into wells (B, bottom; $n=8$ ). (C and $\mathbf{D})$ Flow cytometric analysis from 1 representative experiment (C) and summary data (D) showing the suppression of responder CD8 ${ }^{+}$T cell proliferation after a 6-day IVS, as in $\mathbf{A}$ and B, but in the presence or absence of CD25 hicD127- Tregs or CD25-CD4+ $T$ effector cells (Teffs) isolated from metastatic melanoma (MM) tumor-infiltrating lymphocytes (TILs). $n=15$. Results represent the mean of independent experiments. Horizontal bars depict mean values. Error bars indicate SEM. $P$ values were obtained by repeated-measures ANOVA followed by Tukey's test. ${ }^{*} P<0.05$; ${ }^{* *} P<0.01$; ${ }^{* *} P<0.001$.

CD226 ratio in Tregs correlates with the ex vivo frequency of CD25 $5^{\text {hi } F o x p} 3^{+}$Tregs in the periphery and at tumor sites of MPs. We observed a positive correlation between the CD25 $5^{\text {hiFoxp }} 3^{+}$Treg frequencies and the ratio of TIGIT to CD226 expression (percentage and MFI) in Tregs in MM but not in the periphery (Figure 6A). Because Tregs play a major role in promoting tumor-induced $\mathrm{T}$ cell dysfunction and Treg depletion appears to cooperate with ICB to mediate tumor regression (36-38), we next investigated whether a high TIGIT/CD226 ratio in circulating or Ti Tregs prior to ICB correlated with poor clinical outcome. To this end, we evaluated the TIGIT/CD226 ratios of Tregs in 25 PBMCs and 16 MM samples obtained from MPs prior to ICB, including anti-PD-1 and/or anti-CTLA-4 mAbs. The TIGIT/CD226 
A

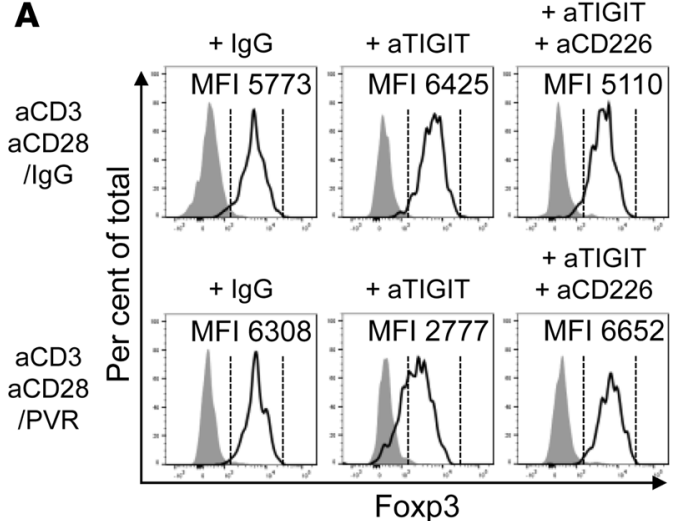

$\square \lg G$

$\square$ aTIGIT

\$ aTIGIT + aCD226

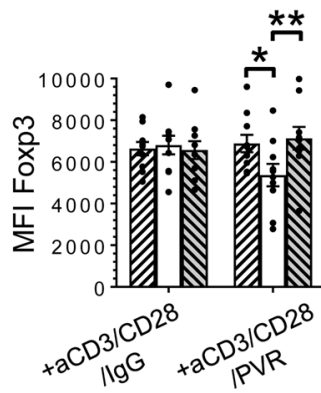

B
$\square \operatorname{lgGbds}+\lg G$
$\square$ PVRbds + IgG
$\square$ PVRbds + aTIGIT
PVRbds + aTIGIT + aCD226
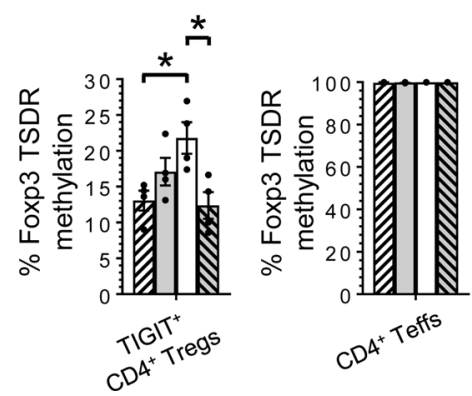

C

$\square \operatorname{lgGbds}+\lg G$

$\square$ PVRbds + IgG

$\square$ PVRbds + aTIGIT

PVRbds + aTIGIT + aCD226
Figure 5. PVR impedes Treg stability through CD226 in melanoma patients. (A) Flow cytometric analysis from 1 representative experiment and pooled data showing Foxp3 expression (mean fluorescence intensity [MFI]) in TIG$\mathrm{IT}^{+}{ }^{+} \mathrm{CD} 25^{\text {hi }}$ CD127- Tregs isolated from PBMCs of melanoma patients (MPs) after a 6-day in vitro stimulation with antiCD3/CD28/IgC or anti-CD3/CD28/PVR beads in the presence of anti-TIGIT and/or anti-CD226-blocking mAbs and/ or IgG control mAbs $n=10$. Solid gray histograms represent Foxp3 expression in CD25-CD4+ Teffs. (B) Summary data showing the percentage of Foxp3 TSDR methylation in TIG$1 T^{+} C D 25^{\text {hi }} C D 127^{-C D 4} 4^{+}$Tregs and $C D 25^{-} C^{-} 4^{+} T$ effector cells (Teffs) isolated from PBMCs of MPs after stimulation as in A. $n=4$. (C) Summary data showing the production of IL-2 by TIGIT ${ }^{+}$CD25 ${ }^{\text {hi }}$ CD127- Tregs isolated from PBMCs of MPs ( $n$ $=12$ ) and total $C D 25^{\text {hiC }}$ D127- Tregs isolated from metastatic melanoma (MM) tumor-infiltrating lymphocytes (TILs) stimulated as in $\mathbf{A}(n=16)$. Results represent the mean of independent experiments. Error bars indicate SEM. $P$ values were obtained by repeated-measures ANOVA followed by Tukey's test. ${ }^{*} P<0.05$; ${ }^{*} P<0.01$; ${ }^{* *} P<0.001$.

ratio in Ti Tregs, but not circulating Tregs, was significantly lower in responders (mean \pm SD, MFI TIGIT/CD226 ratio, $3.8 \pm 2.1)$ than in nonresponders $(10.1 \pm 6.8$ ) (Figure $6 \mathrm{~B})$. The median progression-free survival (PFS) was 12 months for MPs with a TIGIT/CD226 ratio of Ti Tregs < 5.6 (i.e., median of the MFI TIGIT/CD226 ratio in Ti Tregs) and 2 months for MPs with a ratio $>5.6$ (Figure 6C, $P=0.039$ ).

Altogether, our findings show that, in melanoma, a high TIGIT/CD226 ratio in Tregs correlates with high Treg frequencies in tumors and poor clinical outcome upon ICB.

\section{Discussion}

In the present study, we show that Tregs in the periphery and tumors of MPs exhibit a high TIGIT/CD226 expression ratio that regulates their functions and stability. Consistent with previous studies in HDs and mice with melanoma tumors $(12,27)$, we observed that Tregs express high-level TIGIT, with higher expression at tumor sites than in the periphery. As compared with TIGIT- Tregs, TIGIT $^{+}$Tregs exhibited higher suppressive capacities, were more mature/activated, and expressed higher levels of molecules involved in Treg homeostasis, suppressive functions, and stability, including CTLA-4 (13), CD39 (29), PD1 (9), Tim-3 (10 ), Nrp1 (30), and Helios $(33,34)$. They also exhibited higher demethylation of the Foxp3 TSDR, a hallmark of stable and natural Tregs (39-41). In sharp contrast to Teffs, circulating Tregs in HDs and MPs and Ti Tregs displayed lower expression of CD226, which competes with TIGIT for binding to the same ligands, while Ti Tregs further downregulated CD226 as compared with circulating Tregs. Hence and as compared with Teffs, Tregs exhibited an increased TIGIT/CD226 ratio that is higher in tumors than in PBMCs. Strikingly and unlike Teffs, Tregs in PBMCs and tumors of MPs failed to upregulate CD226 expression upon TCR activation, while they upregulated TIGIT, further increasing the TIGIT/CD226 expression ratio. This observation contrasts with the imbalance of TIGIT/ CD226 expression of $\mathrm{CD}^{+}$TILs observed in MM but not in the periphery (16). This striking difference indicates that different mechanisms regulate CD226 expression in Tregs and Teffs. Along these lines, we observed that CD226 expression was decreased at the transcriptional level in Tregs upon TCR activation but not in Teffs, supporting that CD226 gene expression is repressed in activated Tregs in the periphery and at tumor sites. 
A
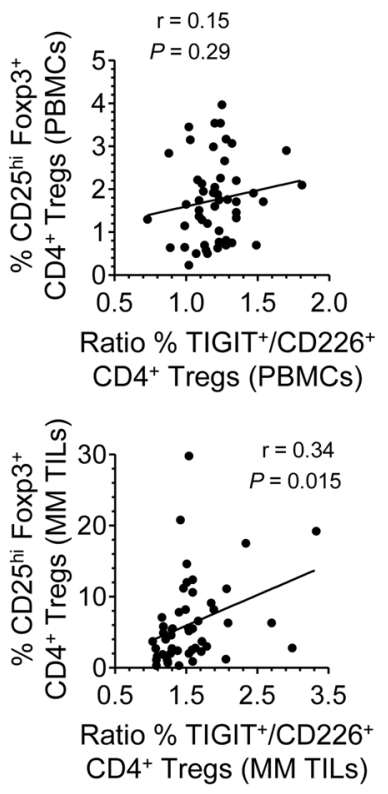

B

- aPD-1
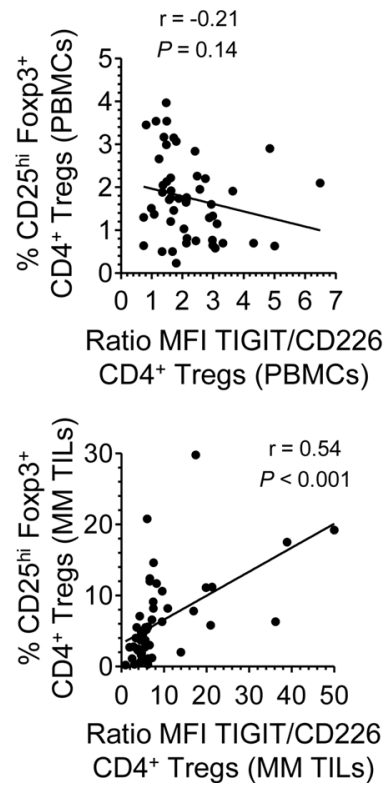

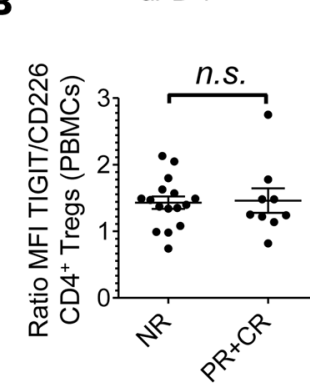

C

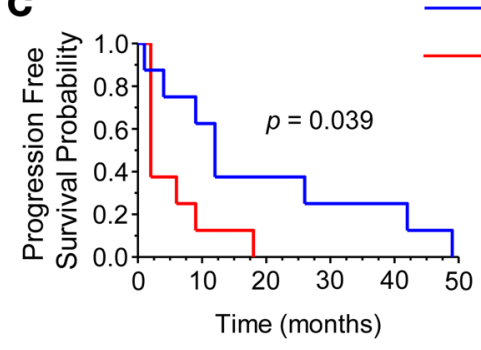

- aPD-1 - aCTLA-4

- $\mathrm{aPD}-1 / \mathrm{aCTLA}-4$

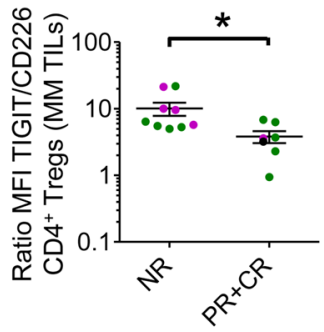

Ratio MFI $<5.6$

Ratio MFI > 5.6

Figure 6. High TIGIT/CD226 imbalance in Tregs in metastatic melanoma correlates with high Treg frequencies and poor clinical outcome upon immune checkpoint blockade. (A) Correlations between the ratio of TIGIT to CD226 expression (percentage and mean fluorescence intensity [MFI]) by CD25 hiFoxp3 ${ }^{+}$

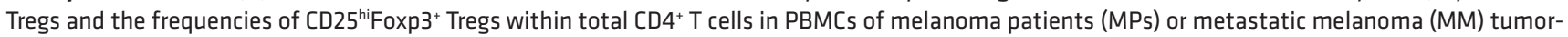
infiltrating lymphocytes (TILs)s. $n=50$. (B) Responses to ICB plotted versus the ratio of TIGIT to CD226 expression (MFI) in CD25 ${ }^{\text {hiF }}$ oxp3 ${ }^{+}$Tregs from PBMCs of MPs treated with aPD-1 mAbs ( $n=25$; left) or MM TILs of MPs treated with aPD- 1 and/or aCTLA-4 mAbs $(n=16$; right). Results represent the mean of independent experiments. Horizontal bars depict mean values. Error bars indicate SEM. (C) Progression-free survival in MPs treated with aPD-1 and/or aCTLA-4 mAbs, stratified by the ratio of TIGIT to CD226 expression (MFI) by tumor-infiltrated CD25 hiFoxp3 ${ }^{+}$Tregs (cutoff value corresponds to median). $n=16$. $P$ values were obtained by Pearson tests $(\mathbf{A})$, unpaired $t$ tests $(\mathbf{B})$, and log-rank test $(\mathbf{C}) .{ }^{*} P<0.05$. ICB, immune checkpoint blockade.

In Tregs, PVR acted on TIGIT and CD226 to augment or counteract Treg-mediated suppression, respectively. The CD226-mediated immunostimulatory effects of PVR were observed in the presence of Tregs isolated either from the periphery or tumor sites and required high-level PVR (i.e., PVR-Fcloaded APCs). In contrast, the TIGIT-mediated immunosuppressive effects of PVR were observed only in the presence of Ti Tregs, and PVR-Fc-loaded APCs, suggesting that they required both high TIGIT and high PVR levels. It is therefore expected that the high TIGIT/CD226 ratio in Ti Tregs cooperates with high-level PVR expressed by tumor cells and APCs in the tumor microenvironment to enhance Treg-suppressive functions. In HDs, FGL2 appears to mediate TIGIT ${ }^{+}$Treg-mediated suppression (12). In line with this study, our findings show that TIGIT ${ }^{+}$Tregs in MPs produced higher FGL2 levels than TIGIT- ${ }^{-}$Tregs upon TCR activation. However, upon CD226 stimulation with PVR (i.e., PVR-Fc plus anti-TIGIT mAbs), Tregs exhibited less suppression but not lower FGL2 production, suggesting that FGL2 is not directly involved in CD226-mediated regulation of Treg functions.

PVR-mediated CD226 activation together with TIGIT blockade decreased Foxp3 expression while increasing Foxp3 TSDR methylation in Tregs of MPs and modestly enhanced their capacity to produce the proinflammatory cytokine IL-2. These findings support the role of PVR in disrupting Treg stability through CD226 by affecting three main features that define Treg stability, including potent suppressive activity, sustained Foxp3 expression, and lack of effector activity (42). In strong support of the CD226-mediated effects of PVR on Treg stability in vivo, we observed a positive correlation between the frequencies of CD25 hiFoxp3 ${ }^{+}$Tregs in MM and the TIGIT/CD226 ratio in Ti Tregs. Such a correlation was not observed in the periphery where PVR levels and TIGIT/CD226 ratio in Tregs are less pronounced. A high TIGIT/ CD226 ratio in Ti Tregs was associated with poor clinical outcome and decreased PFS in MPs treated with ICB, suggesting that the TIGIT/CD226 ratio in Tregs is a marker of Treg stability

In summary, our findings show that Tregs express high-level TIGIT and low-level CD226 as compared with Teffs, resulting in a high ratio of TIGIT/CD226 expression, which is further increased at tumor sites. In sharp contrast to TIGIT, which promotes Ti Treg-mediated suppression, CD226 disrupts Treg-mediated suppression and stability in the periphery and at tumor sites, upon PVR binding. 
Collectively, our findings support that a high TIGIT/CD226 ratio in Tregs together with high PVR expression in the tumor microenvironment promote Treg stability and suppressive functions. They provide the rationale for novel immunotherapies aiming at activating CD226 in Tregs together with TIGIT blockade to counteract Treg suppression patients with solid tumors, including melanoma.

\section{Methods}

Human samples. Blood samples and primary tumor single-cell suspensions were obtained under the University of Pittsburgh Cancer Institute (UPCI) Internal Review Board-approved (IRB-approved) protocols 96-099 and 13-105 from adult patients with stage IV MM. Blood samples from HDs were obtained from the local Central Blood Bank.

Phenotypic analysis and cell sorting. Total $\mathrm{CD}^{+} \mathrm{T}$ cells were purified from samples of patients by magnetic cell separation using MACS Technology (Miltenyi Biotec) and then analyzed ex vivo or sorted by flow cytometry into subsets of Tregs or Teffs for further analysis. The following conjugated mAbs were used in flow cytometric experiments to stain cells either ex vivo or after IVS: CD4-BV510 (clone SK3) or CD8-BV510 (clone SK1) (BD Biosciences); CCR7-FITC (clone 150503, R\&D Systems); CD226-PE (clone 11A8, Biolegend); HLA-DR-ECD (clone Immu-357) or CD45RA-ECD (clone 2H4LDH11LDB9) or CD14-ECD (clone RMO52) and/or CD19-ECD (clone J3-119) (Beckman Coulter); CD39-biotin (clone A1, Biolegend) or CTLA-4-biotin (clone 14D3, Thermo Fisher Scientific) and streptavidin-ECD (Thermo Fisher Scientific); Tim-3-APC (clone 344823, R\&D Systems) or CD155-APC (clone SKII.4, Biolegend); TIGIT-PerCPeFluor710 (clone MBSA43) and CD11c-Alexa700 (clone 3.9) (Thermo Fisher Scientific); PD-1-PECy7 (clone EH12.2H7) or Nrp1-PECy7 (clone 12C2) (BioLegend); and CD25-APCCy7 (clone M-A251, BD Biosciences). Viability of cells was assessed using the LIVE/DEAD Violet Viability/Vitality Kit (Thermo Fisher Scientific). In some experiments, $\mathrm{CD}^{+} \mathrm{T}$ cells were permeabilized after fixation and intracellularly stained with Helios-Pacific Blue (clone 22F6, Biolegend) and/or Foxp3-PE (clone PCH101, Thermo Fisher Scientific) or Foxp3-FITC (clone PCH101) using Foxp3 Staining Buffer Set (Thermo Fisher Scientific). In some experiments, $\mathrm{CD}^{+} \mathrm{T}$ cells were stimulated for 6 days with either with allogeneic nonCD3 cells and $1 \mu \mathrm{g} / \mathrm{ml}$ anti-CD3 mAbs (clone OKT3, Thermo Fisher Scientific) or with anti-CD3/CD28coated MACSiBeads (Miltenyi Biotec) in the presence of agonistic anti-TIGIT mAbs (clone 328.28.2.1; provided by BMS) or IgG control mAbs (BMS), plate-bound at $50 \mathrm{mg} / \mathrm{ml}$, or with Dynabeads M-450 Tosylactivated beads (Thermo Fisher Scientific) coated with anti-CD3 (10\%) and anti-CD28 (10\%) mAbs and either human PVR-Fc construct (provided by BMS) or IgG control Abs (80\%), in the presence of blocking anti-TIGIT (clone 10D7.G8; provided by BMS) and/or anti-CD226 (Abcam; clone DX11) mAbs and/or IgG isotype control mAbs, before phenotypic analysis. Samples were acquired on a FACS LSRII machine (BD Biosciences) and analyzed using Flowjo software v10 (Tree Star). For sorting of CD4 $4^{+} \mathrm{T}$ cell subsets, cells were stained with CD4-BV510, CD127-FITC, and CD25-APCCy7 with or without TIGIT-PerCPeFluor710 and sorted under sterile conditions on a FACSAria machine (BD Biosciences).

CFSE-based proliferation assays. In inhibition of proliferation assays, naive (CD45RA ${ }^{+} \mathrm{CD}^{+} \mathrm{T}$ cells were purified from PBMCs of HDs by magnetic cell separation and stained with CFSE (Thermo Fisher Scientific). CFSE-labeled naive $\mathrm{T}$ cells were cultured for 6 days with autologous non-CD3 cells in the presence or absence of different numbers of $\mathrm{CD} 4^{+} \mathrm{T}$ cells isolated by FACS from PBMCs or TILs of MPs and with $1 \mu \mathrm{g} / \mathrm{ml}$ anti-CD3 mAbs (clone OKT3) and IL-2 (50 IU/ml; Peprotech). In some experiments, cells were stimulated in the presence of $10 \mu \mathrm{g} / \mathrm{ml}$ fully human anti-TIGIT blocking mAbs (clone 10D7.G8) and/or blocking anti-CD226 mAbs (clone DX11) and/or IgG isotype control mAbs, with or without 10 $\mu \mathrm{g} / \mathrm{ml}$ human PVR-Fc construct. After stimulation, cells were stained with CD4-BUV395- (clone RPA-T4, BD Biosciences) as well as CD226-PE-, CD14-ECD- and CD19-ECD-, TIGIT-PerCPeFluor710-, and CD8-APCCy7-conjugated (SK1) (BD Biosciences) mAbs. Viability of cells was assessed during flow cytometric analysis using the LIVE/DEAD Violet Viability/Vitality Kit (Thermo Fisher Scientific).

Multiplex cytokine and ELISA assays. $5 \times 10^{4}$ FACS-sorted CD4 ${ }^{+} \mathrm{T}$ cells were incubated for 6 days in 96-well plates containing $200 \mu \mathrm{l}$ culture medium with same number of anti-CD3/CD28 MACSiBeads. In some experiments, $\mathrm{CD}^{+} \mathrm{T}$ cells were incubated with same number of Dynabeads $\mathrm{M}-450$ Tosylactivated beads coated with anti-CD3 (10\%) and anti-CD28 (10\%) mAbs and either human PVR-Fc construct or IgG control Abs (80\%) in the presence of blocking anti-TIGIT (10D7.G8) and/or anti-CD226 (DX11) mAbs and/or IgG isotype control mAbs. Cytokine production in culture supernatants was determined using the Human TH17 Magnetic Bead Panel (HT17MG-14K-10; EMD Millipore) and TGF- $\beta$ 1, 2 , 3 Magnetic 
Bead Panel (TGFBMAG-64K-03; EMD Millipore) according to manufacturers' instructions. FGL2 production in culture supernatants was assessed by ELISA using the LEGEND MAX Human FGL2 ELISA Kit (Biolegend) according to the manufacturer's instructions.

Quantitative PCR. $5 \times 10^{5}$ FACS-sorted CD4 ${ }^{+}$T cells were stimulated for 6 days with an equal number of anti-CD3/CD28-coated MACSiBeads. RNA was extracted with RNeasy Mini Kits (Qiagen), and cDNA was prepared by reverse transcription using M-MLV Reverse Transcriptase (Thermo Fisher Scientific). RT-PCR was performed with StepOne System (Thermo Fisher Scientific). All samples were normalized to the expression of $\beta$-glucuronidase ( $\beta$-Gus). TIGIT expression was detected using previously described primers and probes (11). CD226 expression was detected using the following forward $(\mathrm{F})$ and reverse $(\mathrm{R})$ primers and probe (P): F (5'-CAGCACTCACATCTCAAGAACC-3'), R (5'-TGTTCTCGGCAAAGGGAACT-3'), and P (5'-FAM-ACAGAGCTCTATGTGAAGAGGTGCTTTGGC-3'BHQ).

Foxp3 TSDR methylation analysis. FACS-sorted $\mathrm{CD}^{+} \mathrm{T}$ cells were either analyzed ex vivo or after IVS with equal number of anti-CD3/CD28 Dynabeads coated with either human PVR-Fc construct or IgG control Abs in the presence of anti-TIGIT (clone 10D7.G8) and/or anti-CD226 (clone DX11) mAbs and/ or IgG isotype control mAbs. Methylation analysis of the TSDR region within the first intron of the Foxp3 gene locus was performed on genomic DNA extracted from CD4 ${ }^{+} \mathrm{T}$ cells as previously described (43).

Statistics. The normality of each variable was evaluated using the Shapiro-Wilk test. Statistical comparison between two groups was assessed by 2 -tailed paired or unpaired $t$ tests. For multiple group comparison, in cases of normally distributed data, statistical significance was assessed by a 1-way or repeated-measures ANOVA for independent and dependent data, respectively, followed by Tukey's multiple comparisons test. In cases of not normally distributed data, the comparison of variables was performed with a Kruskal-Wallis test or a Friedman test for unpaired and paired data, respectively, followed by a Dunn's multiple comparisons test. For correlation studies, $P$ values were obtained by Pearson tests. PFS was estimated by Kaplan-Meier method and compared using the log-rank test. $P$ values of less than 0.05 were considered significant. Statistical analysis was performed using GraphPad Prism 5.

Study approval. All studies using human tissues and primary human cells were approved by the IRB of UPCI. Written informed consent was received from patients prior to their inclusion in the study.

\section{Author contributions}

JF designed the study, performed experiments, analyzed data, and wrote the manuscript. ZS performed quantitative PCR and Foxp3 TSDR methylation analysis. JMC, MK, OP, CM, and RA processed samples and contributed to data interpretation. SS provided technical assistance with experiments and contributed to data interpretation. CS helped acquire samples. JMK managed patients and provided clinical data. DD managed patients and provided and analyzed clinical data. AJK provided reagents and contributed to data interpretation. HMZ designed and supervised the study and wrote the manuscript. All of the authors reviewed the manuscript before submission.

\section{Acknowledgments}

This work was supported by the Office of the Assistant Secretary of Defense for Health Affairs through the Peer-Reviewed Cancer Research Program under award W81XWH-15-1-0510 (to JF). Opinions, interpretations, conclusions, and recommendations are those of the author and are not necessarily endorsed by the Department of Defense. The US Army Medical Research Acquisition Activity is the awarding and administering acquisition office. This work was also supported by a National Cancer Institute (NCI) career development award (P50 CA121973) (to JF), NIH/NCI grants R01CA157467 and R01CA228181 (to HMZ), NCI grant P50CA121973 (to JMK), a research grant from Bristol-Myers Squibb (to HMZ), and a cancer vaccine collaborative clinical strategy team grant (to HMZ). This project used the Luminex core of the UPMC Hillman Cancer Center, which is supported in part by NIH award P30CA047904 and the Flow Facility of the Department of Immunology, University of Pittsburgh.

Address correspondence to: Hassane M. Zarour or Julien Fourcade, UPMC Hillman Cancer Center, Research Pavilion, Suite 1.32a, 5117 Centre Avenue, Pittsburgh, Pennsylvania 15213-2582, USA. Phone: 412.623.3272; Email: zarourhm@upmc.edu (H.M. Zarour). Phone: 412.623.3244; Email: fourcadejj@ upmc.edu (J. Fourcade). 
1. Sakaguchi S, Yamaguchi T, Nomura T, Ono M. Regulatory T cells and immune tolerance. Cell. 2008;133(5):775-787.

2. Sakaguchi S. Naturally arising Foxp3-expressing CD25+CD4+ regulatory T cells in immunological tolerance to self and nonself. Nat Immunol. 2005;6(4):345-352.

3. Ladányi A, et al. FOXP3+ cell density in primary tumor has no prognostic impact in patients with cutaneous malignant melanoma. Pathol Oncol Res. 2010;16(3):303-309.

4. Mourmouras V, et al. Evaluation of tumour-infiltrating CD4+CD25+FOXP3+ regulatory T cells in human cutaneous benign and atypical naevi, melanomas and melanoma metastases. Br J Dermatol. 2007;157(3):531-539.

5. Jandus C, Bioley G, Speiser DE, Romero P. Selective accumulation of differentiated FOXP3(+) CD4 (+) T cells in metastatic tumor lesions from melanoma patients compared to peripheral blood. Cancer Immunol Immunother. 2008;57(12):1795-1805.

6. Ahmadzadeh M, et al. FOXP3 expression accurately defines the population of intratumoral regulatory T cells that selectively accumulate in metastatic melanoma lesions. Blood. 2008;112(13):4953-4960

7. Gerber AL, et al. High expression of FOXP3 in primary melanoma is associated with tumour progression. Br J Dermatol. 2014;170(1):103-109.

8. Cesana GC, et al. Characterization of CD4+CD25+ regulatory T cells in patients treated with high-dose interleukin-2 for metastatic melanoma or renal cell carcinoma. J Clin Oncol. 2006;24(7):1169-1177.

9. Francisco LM, et al. PD-L1 regulates the development, maintenance, and function of induced regulatory T cells. $J$ Exp Med. 2009;206(13):3015-3029.

10. Sakuishi K, et al. TIM3+FOXP3+ regulatory T cells are tissue-specific promoters of T-cell dysfunction in cancer. Oncoimmunology. 2013;2(4):e23849.

11. Yu X, et al. The surface protein TIGIT suppresses $\mathrm{T}$ cell activation by promoting the generation of mature immunoregulatory dendritic cells. Nat Immunol. 2009;10(1):48-57.

12. Joller N, et al. Treg cells expressing the coinhibitory molecule TIGIT selectively inhibit proinflammatory Th1 and Th17 cell responses. Immunity. 2014;40(4):569-581.

13. Wing K, et al. CTLA-4 control over Foxp3+ regulatory T cell function. Science. 2008;322(5899):271-275.

14. Stanietsky N, et al. The interaction of TIGIT with PVR and PVRL2 inhibits human NK cell cytotoxicity. Proc Natl Acad Sci USA. 2009;106(42):17858-17863.

15. Pende D, et al. PVR (CD155) and Nectin-2 (CD112) as ligands of the human DNAM-1 (CD226) activating receptor: involvement in tumor cell lysis. Mol Immunol. 2005;42(4):463-469.

16. Chauvin JM, et al. TIGIT and PD-1 impair tumor antigen-specific CD8 ${ }^{+} \mathrm{T}$ cells in melanoma patients. J Clin Invest. 2015;125(5):2046-2058.

17. Shibuya A, et al. DNAM-1, a novel adhesion molecule involved in the cytolytic function of T lymphocytes. Immunity. 1996;4(6):573-581.

18. Bottino C, et al. Identification of PVR (CD155) and Nectin-2 (CD112) as cell surface ligands for the human DNAM-1 (CD226) activating molecule. J Exp Med. 2003;198(4):557-567.

19. Tahara-Hanaoka S, et al. Functional characterization of DNAM-1 (CD226) interaction with its ligands PVR (CD155) and nectin-2 (PRR-2/CD112). Int Immunol. 2004;16(4):533-538.

20. Shibuya K, et al. CD226 (DNAM-1) is involved in lymphocyte function-associated antigen 1 costimulatory signal for naive T cell differentiation and proliferation. J Exp Med. 2003;198(12):1829-1839.

21. Joller N, et al. Cutting edge: TIGIT has T cell-intrinsic inhibitory functions. J Immunol. 2011;186(3):1338-1342.

22. Lozano E, Dominguez-Villar M, Kuchroo V, Hafler DA. The TIGIT/CD226 axis regulates human T cell function. J Immunol. 2012;188(8):3869-3875.

23. Johnston RJ, et al. The immunoreceptor TIGIT regulates antitumor and antiviral CD8(+) T cell effector function. Cancer Cell. 2014;26(6):923-937.

24. Iguchi-Manaka A, et al. Accelerated tumor growth in mice deficient in DNAM-1 receptor. J Exp Med. 2008;205(13):2959-2964.

25. Ramsbottom KM, et al. Cutting edge: DNAX accessory molecule 1-deficient CD8+ T cells display immunological synapse defects that impair antitumor immunity. J Immunol. 2014;192(2):553-557.

26. Zhang Y, et al. Genome-wide DNA methylation analysis identifies hypomethylated genes regulated by FOXP3 in human regulatory T cells. Blood. 2013;122(16):2823-2836.

27. Kurtulus S, et al. TIGIT predominantly regulates the immune response via regulatory T cells. J Clin Invest. 2015;125(11):4053-4062.

28. Fuhrman CA, et al. Divergent phenotypes of human regulatory T cells expressing the receptors TIGIT and CD226. J Immunol. 2015;195(1):145-155.

29. Borsellino G, et al. Expression of ectonucleotidase CD39 by Foxp3+ Treg cells: hydrolysis of extracellular ATP and immune suppression. Blood. 2007;110(4):1225-1232.

30. Delgoffe GM, et al. Stability and function of regulatory T cells is maintained by a neuropilin-1-semaphorin-4a axis. Nature. 2013;501(7466):252-256.

31. Kim HJ, et al. Stable inhibitory activity of regulatory T cells requires the transcription factor Helios. Science. 2015;350(6258):334-339.

32. Nakagawa H, Sido JM, Reyes EE, Kiers V, Cantor H, Kim HJ. Instability of Helios-deficient Tregs is associated with conversion to a T-effector phenotype and enhanced antitumor immunity. Proc Natl Acad Sci USA. 2016;113(22):6248-6253.

33. Miyara M, et al. Functional delineation and differentiation dynamics of human CD4+ T cells expressing the FoxP3 transcription factor. Immunity. 2009;30(6):899-911.

34. Zhou L, Chong MM, Littman DR. Plasticity of CD4+ T cell lineage differentiation. Immunity. 2009;30(5):646-655

35. Sawant DV, Vignali DA. Once a Treg, always a Treg? Immunol Rev. 2014;259(1):173-191.

36. Arce Vargas F, et al. Fc-optimized anti-CD25 depletes tumor-infiltrating regulatory $\mathrm{T}$ cells and synergizes with PD-1 blockade to eradicate established tumors. Immunity. 2017;46(4):577-586.

37. Goding SR, et al. Restoring immune function of tumor-specific CD4+ T cells during recurrence of melanoma. J Immunol. 2013;190(9):4899-4909.

38. Quezada SA, Peggs KS, Simpson TR, Shen Y, Littman DR, Allison JP. Limited tumor infiltration by activated T effector cells 
restricts the therapeutic activity of regulatory T cell depletion against established melanoma. J Exp Med. 2008;205(9):2125-2138.

39. Floess S, et al. Epigenetic control of the foxp3 locus in regulatory T cells. PLoS Biol. 2007;5(2):e38.

40. Baron U, et al. DNA demethylation in the human FOXP3 locus discriminates regulatory T cells from activated FOXP3(+) conventional T cells. Eur J Immunol. 2007;37(9):2378-2389.

41. Waight JD, et al. Cutting edge: epigenetic regulation of Foxp3 defines a stable population of CD4+ regulatory T cells in tumors from mice and humans. J Immunol. 2015;194(3):878-882.

42. Overacre AE, Vignali DA. T(reg) stability: to be or not to be. Curr Opin Immunol. 2016;39:39-43.

43. Fourcade J, et al. Human tumor antigen-specific helper and regulatory T cells share common epitope specificity but exhibit distinct T cell repertoire. J Immunol. 2010;184(12):6709-6718. 